\title{
The mineralogy and space weathering of a regolith grain from 25143 Itokawa and the possibility of annealed solar wind damage
}

\author{
Dennis Harries ${ }^{*}$ and Falko Langenhorst
}

\begin{abstract}
We report the results of detailed mineralogical investigations by analytical scanning and transmission electron microscopy of particle RA-QD02-0115 recovered from the surface of asteroid 25143 Itokawa. We divided the 65 $\mu \mathrm{m} 50 \mu \mathrm{m}$ small particle into eight individual subsample slices via the focused ion beam method. The particle dominantly consists of olivine and contains inclusions of merrillite, tetrataenite/taenite, troilite, chromite, kamacite, and $\mathrm{Cl}$-bearing apatite (in approx. decreasing order of frequency). The composition of olivine (fayalite $29.81 .1 \mathrm{~mol}$ $\%$ and molar Fe/Mn ratio of 57 2) as well as the Ni-rich metal assemblage indicates an LL-type affinity in accord with previous classifications. The particle shows effects of solar wind irradiation on one of its principal faces. Olivine developed an approximately $34 \mathrm{~nm}$ wide rim composed of low-angle misoriented, nanometer-sized crystallites accompanied by a small amount of amorphous material. Exposed troilite developed a 4 to $8 \mathrm{~nm}$ wide polycrystalline rim with large-angle misorientations of the iron sulfide nanocrystallites. Merrilite shows marginally discernable surface damage but was too unstable under the electron beam for a detailed study. Cl-bearing apatite was found fully crystalline with no discernable rim structure. We discuss the unusual polycrystalline nature of the olivine rim in terms of possible annealing and recrystallization effects, which may have occurred during periods of time when Itokawas surface temperature may have been warmer due to closer perihelion distances. Model calculations show that the dynamical orbital evolution of near-Earth asteroids could lead to complex space weathering processes, arising from the competing interplay between irradiation-induced damaging and thermally driven annealing.
\end{abstract}

Keywords: Hayabusa; Itokawa; Olivine; Troilite; Phosphates; Space weathering; Orbital evolution; Irradiation; Annealing; Recrystallization

\section{Findings}

\section{Background}

The Hayabusa spacecraft returned for the first time pristine surface material from a small airless body of the Solar System, namely from the S-type asteroid 25143 Itokawa (Nakamura et al. 2011, Noguchi et al. 2011). After the Moon s regolith this is only the second time that material was directly sampled on the surface of an extraterrestrial body.

In analogy to the lunar soil it was originally assumed that Hayabusa dust grains were affected by space weathering (Chapman 2004). Space weathering is a fundamental

\footnotetext{
* Correspondence: Dennis.Harries@uni-jena.de

Analytical Mineralogy of Micro- and Nanostructures, Institute of Geoscience, Friedrich Schiller University Jena, Carl-Zeiss-Promenade 10, 07745 Jena,
} Germany process on airless bodies that causes modifications of mineral surfaces via (i) irradiation by cosmic rays and, in particular, by solar wind ions and/or (ii) via impacts of micrometeorites. Such a bombardment with high-energy particles can result in multiple effects: amorphization, sputtering/redepositioning, fracturing, melting, and vaporization of surface material. The complexities of space-weathered mineral surfaces were first studied on lunar samples, showing the formation of thin (50 $200 \mathrm{~nm}$ ) amorphous silicate layers that often contain nanometer-sized particles of Fe metal (Keller and McKay 1997). This nanophase Fe (npFe $\left.{ }^{0}\right)$ is considered to alter the spectral properties of asteroid surfaces by reducing the albedo and characteristic mineral absorption bands as well as by reddening the surface reflectance spectra (Pieters et al. 2000). These optical effects explain the apparent mismatch between the reflectance

\section{穴 Springer}


spectra of S-type asteroids and those of ordinary chondrites (Binzel et al. 1996).

Recent investigations of Hayabusa dust grains revealed indeed that some of them contain similar space weathering effects as lunar soil samples (Nakamura et al. 2012; Noguchi et al. 2011, 2014). The internal structure of the damaged surface layers can be quite complex and may consist of various amorphous layers with embedded $n p F e^{0}$. Based on detailed transmission electron microscopy (TEM) observations, it was concluded that solar wind irradiation and ion implantation are the major causes for the observed space weathering in Hayabusa dust grains (Noguchi et al. 2014).

In this study, we present the results of an analytical TEM study on an olivine-dominated Hayabusa dust grain (RA-QD02-0115), which was one of two grains (the other one was RB-QD04-0042) that were provided to us in the context of the first international announcement of opportunity for Hayabusa sample investigation (Yada et al. 2014). RA-QD02-0115 turned out to contain a rich mineral assemblage. Here, we will focus on the space weathering effects observed on this particular sample and their possible implications for space weathering on near-Earth asteroids. The metallography and petrogenetic history of the particle will be the scope of a separate publication.

\section{Methods}

The surface morphology and exposed mineral grains of particle RA-QD02-0115 were first inspected by means of scanning electron microscopy (SEM) and energy-dispersive X-ray spectroscopy (EDX) using a FEI Quanta3D FEG dual beam focused ion beam (FIB)-SEM workstation (FEI, Hillsboro, OR, USA). Preparation followed the procedure described in Langenhorst et al. (2014), with the exception that an additional platinum sheet was used as support for the grain.

Our planning of the sections aimed at sampling all principle types of mineral inclusions observed during initial SEM study. We were able to subdivide the particle into eight slices, which were individually mounted to post-type copper TEM grids. Four of the eight subsamples (designations RA-QD02-0115-02, -03, -04, -05) were thinned to electron transparency using successively lower $\mathrm{Ga}^{+}$ion beam currents of $5 \mathrm{nA}, 1 \mathrm{nA}$, and $300 \mathrm{pA}$ at $30 \mathrm{keV}$ energy. Final polishing was done using a current of 77 to 150 $\mathrm{pA}$ at $5 \mathrm{keV}$ ion energy. The remaining slices were preserved for future studies at thicknesses of 2 to $3 \mu \mathrm{m}$.

The electron transparent subsamples were studied by TEM using a FEI Tecnai $G^{2}$ FEG installed at the Institute for Geosciences, University of Jena, Germany. The microscope is equipped with an Oxford $80 \mathrm{~mm}^{2}$ energydispersive SDD X-ray detector and a Gatan UltraScan $2 \mathrm{k}$ CCD camera (Gatan Inc., Pleasanton, CA, USA). It was operated at $200 \mathrm{kV}$ acceleration voltage. EDX analyses were quantified according to the Cliff-Lorimer technique involving an absorption correction based on charge neutrality (van Cappellen and Doukhan 1994). Cliff-Lorimer k factors for the elements $\mathrm{O}, \mathrm{Mg}, \mathrm{Si}, \mathrm{Mn}$, and Fe were determined using an olivine sample previously characterized by WDS-EPMA. Other $\mathrm{k}$ factors were interpolated using theoretical $\mathrm{k}$ factors calculated with FEIs TIA software.

\section{Results}

\section{Characteristics and mineralogy of particle RA-QD02-0115}

RA-QD02-0115 was received as an oblate particle with lateral dimensions of $65 \mu \mathrm{m} 50 \mu \mathrm{m}$ (Figure 1A) and a thickness of 10 to $12 \mu \mathrm{m}$ (measured on FIB cross sections, Figure 1B). The initial study by SEM showed that the particle is an angular fragment with only very slight rounding of its edges. The bulk of the particle is composed of olivine with several mineral inclusions and voids exposed at its natural surface. The two most prominent inclusions were identified by SEM-EDX as Fe sulfide $(10 \mu \mathrm{m})$ and a composite inclusion of $\mathrm{Fe}$ sulfide and $\mathrm{Fe}-\mathrm{Ni}$ metal $(5 \mu \mathrm{m})$. Minor inclusions were identified as merrillite, Fe-Ni metal, Fe sulfide (likely troilite), and chromite. One exposed inclusion was found to be a chlorine-bearing apatite (confirmed by TEM-selected area electron diffraction (SAED)). Empty voids within olivine at the particles surface showed polyhedral internal surfaces but contained no identifiable residues of any former fluid phase. Small-sized $(<1 \mu \mathrm{m})$ splash form objects (apparent droplets) were rarely observed across the visible surface.

Mineral inclusions detected during FIB sectioning are listed in Table 1. In particular, merrillite was encountered frequently. Several FIB slices and the material removed during the approaches to the final thicknesses of the slices showed that in many cases, the phosphate grains were directly associated with metal grains of similar sizes. Notably, phosphate and metal grains were prevalent on one side of the particle (and often in contact) whereas the other side had prevalently troilite inclusions. Details of the mineralogy of those subsamples thinned to electron transparency and studied by TEM are described in the following.

Olivine TEM-EDX analysis of the host olivine resulted in a composition of $\mathrm{Fa}_{29.81 .1}$ and a molar $\mathrm{Fe} / \mathrm{Mn}$ ratio of 57 2 (95\% confidence). Both parameters suggest an LL-type material as indicated by previous study of Itokawa particles (Nakamura et al. 2011). No significant heterogeneity was detected among different subsamples of the particle.

Chromite Chromite encountered in subsamples 03 and 05 contains minor amounts of aluminum and magnesium, yielding the empirical formula $\left(\mathrm{Fe}_{0.86} \mathrm{Mg}_{0.08} \mathrm{Mn}_{0.06}\right)$ $\left(\mathrm{Cr}_{1.59} \mathrm{Al}_{0.32} \mathrm{Fe}_{0.11}\right) \mathrm{O}_{4}$ (based on interpolated $\mathrm{k}$ factors for 

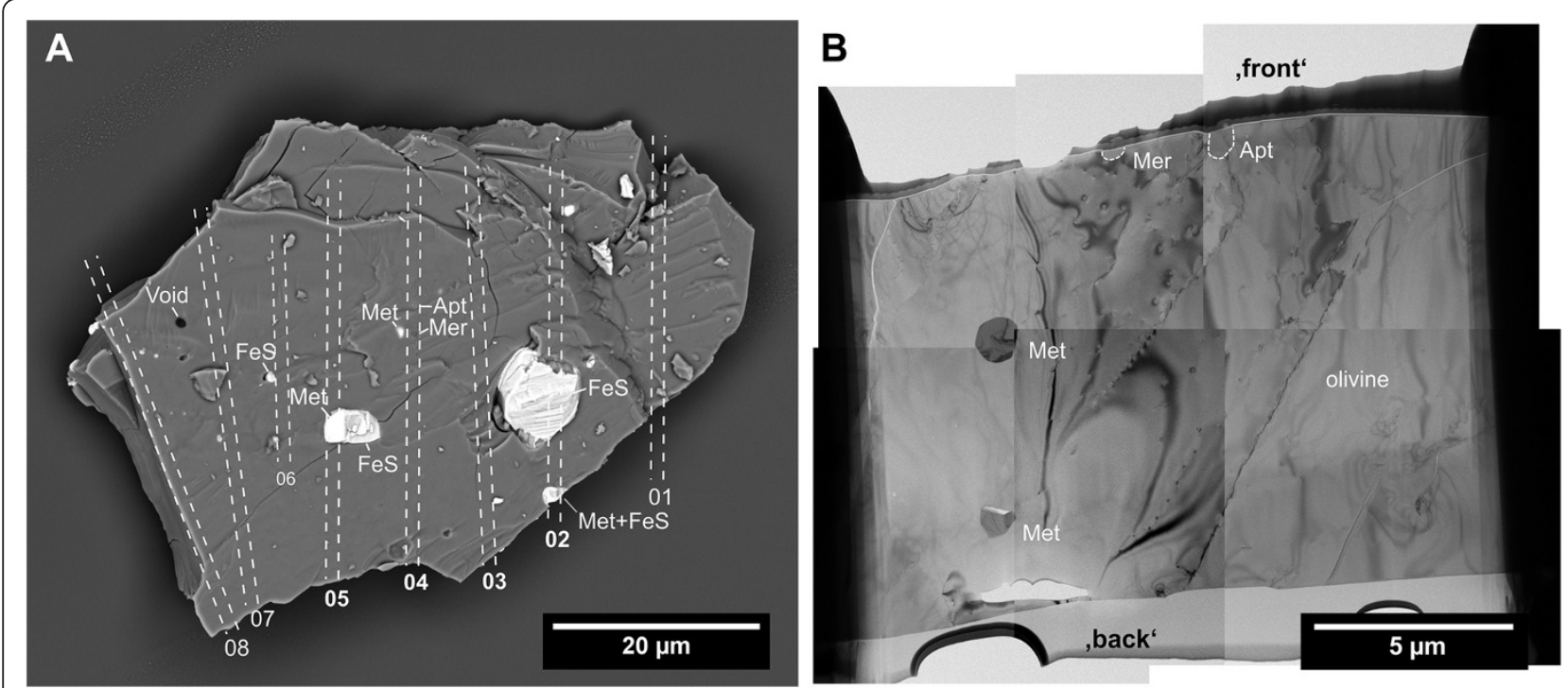

Figure 1 Overview of RA-QD02-0115. (A) SEM backscatter electron image of the front side of the particle. FIB sections are indicated by their subsample number. (B) TEM bright-field image montage of subsample 04.

$\mathrm{Al}$ and $\mathrm{Cr}, \mathrm{Mn}$ and therefore the content of $\mathrm{Fe}^{3+}$ may be overestimated due to the overlap of $\mathrm{Cr} K \beta$ on $\mathrm{Mn} \mathrm{K} \alpha$ ). In subsample 03, a large (approximately $5 \mu \mathrm{m}$ ) chromite grain is associated with a cavity of similar size.

Phosphates TEM-EDX analyses of merrillite showed characteristic contents of magnesium and sodium, but quantification was not attempted due to the instability of this mineral during electron irradiation; it was, however, positively identified by SAED. The same applies to the only grain of chlorine-bearing apatite initially observed at the particles surface and sliced to electron transparency in subsample 04. TEM-EDX analysis showed only a minor chlorine peak (molar $\mathrm{Cl} / \mathrm{P} \approx 0.03$ ) after about 5 min of irradiation at $200 \mathrm{keV}$ (TEM imaging and SAED were given priority for the study of possible space weathering effects, see below), although SEM-EDX indicated a considerable $\mathrm{Cl}$ content before $\mathrm{FIB}$ preparation (molar $\mathrm{Cl} / \mathrm{P} \approx 0.10$ ). No fluorine, sulfur, or any other possibly substituting elements were detected.

Fe sulfide The iron sulfide in slices 02 and 05 was identified by SAED as troilite. Besides Fe and S, no other elements were detected by EDX analysis above the respective detection limits (approx. 0.1 wt.\% for $\mathrm{Cr}$ and $\mathrm{Ni}$ ).

Metal Metallic inclusions comprise kamacite, taenite, and, particularly frequently, tetrataenite (all identified by SAED, tetrataenite via its superstructure spots). Metal grains are normally composed of a single phase or consist of two (rarely more) subgrains of different metal phases. In subsample 05, a large metal sulfide composite grain occurs at the particles surface. The surface-exposed metal part consists of a kamacite and a taenite subgrain. Two metal grains in subsample 4 are associated with Fe carbides (Harries and Langenhorst 2014).

Table 1 Mineral inclusions encountered in RA-QD02-0115 during FIB preparation (number of grains in brackets)

\begin{tabular}{|c|c|c|}
\hline Slice number & Minerals detected & Remarks/largest dimensions \\
\hline 01 & Merrillite (10), troilite (1) & Merrillite grains size 0.2 to $0.9 \mu \mathrm{m}$ \\
\hline 02 & Merrillite (4), metal (2), troilite (2) & Large troilite grain (approximately $8 \mu \mathrm{m}$ ) \\
\hline 03 & Metal (4), merrillite (3), chromite (1). & $\begin{array}{l}\text { Chromite up to approximately } 5 \mu \mathrm{m} \text {, two metal grains }(1.3 \text { to } 1.4 \mu \mathrm{m}) \text { with } \\
\text { adjacent } \\
\text { merrillite grains ( } 1.1 \text { to } 1.3 \mu \mathrm{m} \text {, other merrillites } 0.6 \text { to } 0.9 \mu \mathrm{m})\end{array}$ \\
\hline 04 & Metal (5), merrillite (4), chlorapatite (1), troilite (1) & Three metal grains (0.5 to $1.3 \mu \mathrm{m})$ with adjacent merrillite grains (0.5 to $0.8 \mu \mathrm{m})$ \\
\hline 05 & Troilite (2), merrillite (1), metal (1), chromite (1) & $\begin{array}{l}\text { Troilite up to approximately } 3 \mu \mathrm{m} \text { in contact with metal }(0.8 \text { to } 2.4 \mu \mathrm{m}) \text {. Chromite } \\
(0.6 \mu \mathrm{m}) \text { in contact with troilite }(1.2 \mu \mathrm{m})\end{array}$ \\
\hline 06 & Troilite (2), chromite (1) & Troilite $(0.7 \mu \mathrm{m})$ in contact with chromite $(0.5 \mu \mathrm{m})$ \\
\hline 07 & Troilite (>5), chromite (1) & Troilite up to $1 \mu \mathrm{m}$, others $<0.5 \mu \mathrm{m}$ \\
\hline 08 & Troillite $(>10)$ & Troilite up to $1.5 \mu \mathrm{m}$, others $<0.5 \mu \mathrm{m}$ \\
\hline
\end{tabular}


Defect microstructures of olivine in particle RA-QD02-0115 The bulk olivine in RA-QD02-0115 is polycrystalline with frequent subgrain (low-angle) boundaries and rarer largeangle grain boundaries (subgrain sizes approximately 1 to $10 \mu \mathrm{m})$. Only one olivine subgrain (Figure 2) shows an enhanced dislocation density (approx. $10^{12} \mathrm{~m}^{-2}$ ). We used TEM dark field imaging under various diffraction conditions to identify the Burgers vector of these dislocations. The diffraction vector $\mathbf{g}=[110]$ yielded out-of-contrast conditions and thus points to a Burgers vector of $\mathbf{b}=$ [001]. The orientation of the dislocation lines relative to the olivine lattice indicates them to be roughly parallel to the [001] direction and, therefore, mainly of the screw type. However, the slight curvature of the dislocation lines across the subgrain suggests an additional but minor edge-type contribution to the dislocations.

\section{Space weathering of particle RA-QD02-0115}

Olivine in all four subsamples studied by TEM shows a rim on one of the principal surfaces (Figure $3 \mathrm{~A}, \mathrm{~B}, \mathrm{C}$; the front side as shown in Figure $1 \mathrm{~B}$ ) of the platy particle, but not on the other (the back side which could not be imaged by SEM). The rim has an average thickness of $344 \mathrm{~nm}$ and shows only very small local variations in thickness. The rim consists predominantly of nanocrystallites of olivine that show misorientations relative to the underlying single crystal by up to 3 as indicated by high-resolution transmission electron microscopy (HRTEM) images (Figure 3D,E). The domain size of the

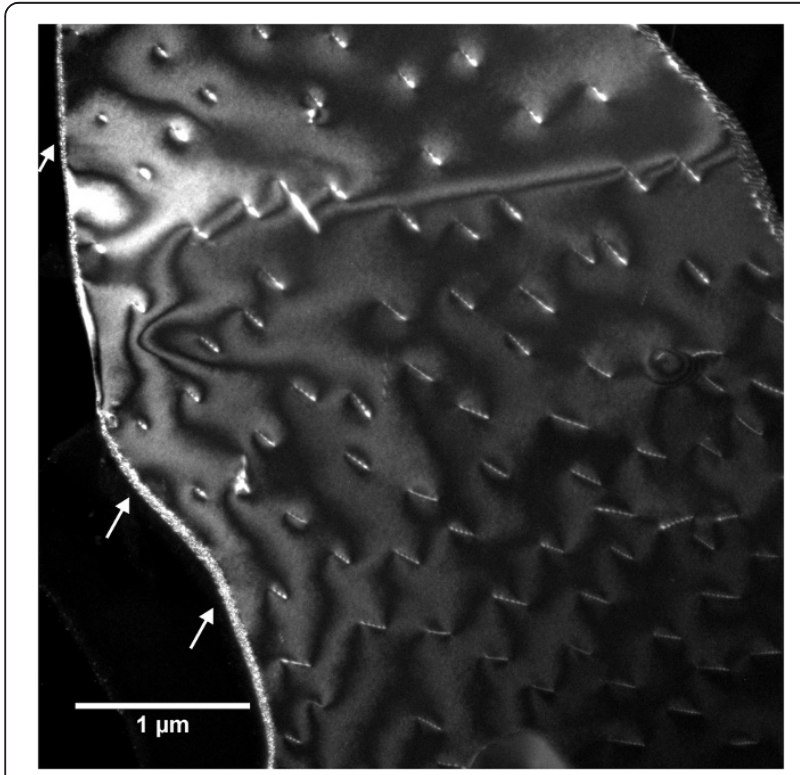

Figure 2 Weak-beam dark-field TEM image of an olivine subgrain in subsample 03. A high density of mixed screw- and edge-type [001] dislocations $(g=222)$ is visible. Also visible is the strongly diffracting nanocrystalline olivine rim at the surface of the particle (arrows). nanocrystallites is approximately 3 to $10 \mathrm{~nm}$ based on HR-TEM and TEM dark field images (Figure 3C). These images also suggest that the rim is predominantly crystalline with only a small contribution of amorphous material. Although no aperodic domains are directly visible in HR-TEM images, an amount of up to 40 vol.\% of amorphous material may be hidden by the remaining crystalline material, due to the physics of contrast formation in HR-TEM images (Wang et al. 1993). However, from dark field images showing strong diffraction, we estimated the amount to be very low, probably around 10 vol.\%. TEM-EDX maps and extracted elemental profiles show no compelling changes in the chemical composition of the rim relative to the bulk olivine. An increase in molar $\mathrm{Si} / \mathrm{Mg}$ in the outer $30 \mathrm{~nm}$ may be just resolvable but this requires a strong thickness correction (the thickness changes by about $100 \mathrm{~nm}$ along the 130-nm rim profile, probably due to different sputtering yields of the Pt cover and the silicate). We did not detect $n p F e^{0}$ in the rim. Only very few, weakly contrasting solar flare tracks have been detected, despite the large surface areas of the FIB sections. The track density is estimated to be about $10^{7}$ to $10^{8} \mathrm{~cm}^{-2}$.

Because the whole front side of the particle was coated with a $30 \mathrm{~nm}$ thick carbon layer prior to the main SEM study and was additionally protected by an electron beam deposited layer of platinum before any ion beam irradiation occurred, we confidently exclude that this olivine surface layer was produced by incidence of $\mathrm{Ga}^{+}$ ions. Moreover, the nanocrystalline rim continues under the shadowing olivine particle shown in Figure 3A, indicating that the rim existed before the small particle got attached to the surface, which likely happened during sample collection on Itokawa or handling during recovery. It also appears unlikely that an originally amorphous rim would have been turned nanocrystalline during FIB thinning as heavy ion irradiation generally generates the opposite effect. A similar rim was also detected in a sample sliced by ultramicrotomy (Keller and Berger 2014), practically ruling out a FIB preparation artifact. During TEM observation, we did not detect any change in the structural state of the rims.

The merrilite and chlorine-bearing apatite grains of subsample 04 (Figure 4; both identified by SAED and EDX) were investigated first in our TEM observations of this FIB slice in order to minimize any effects from prolonged electron irradiation. Both grains were originally exposed at the front surface of the particle. The merrilite showed at the earliest observation a very weakly contrasting, gradual rims structure (roughly $50 \mathrm{~nm}$ wide, Figure 4A), which, however, could not be investigated in greater detail, because the merrillite rapidly deteriorated under the electron beam and formed abundant vesicles. The apatite grain was found to be fully crystalline with 


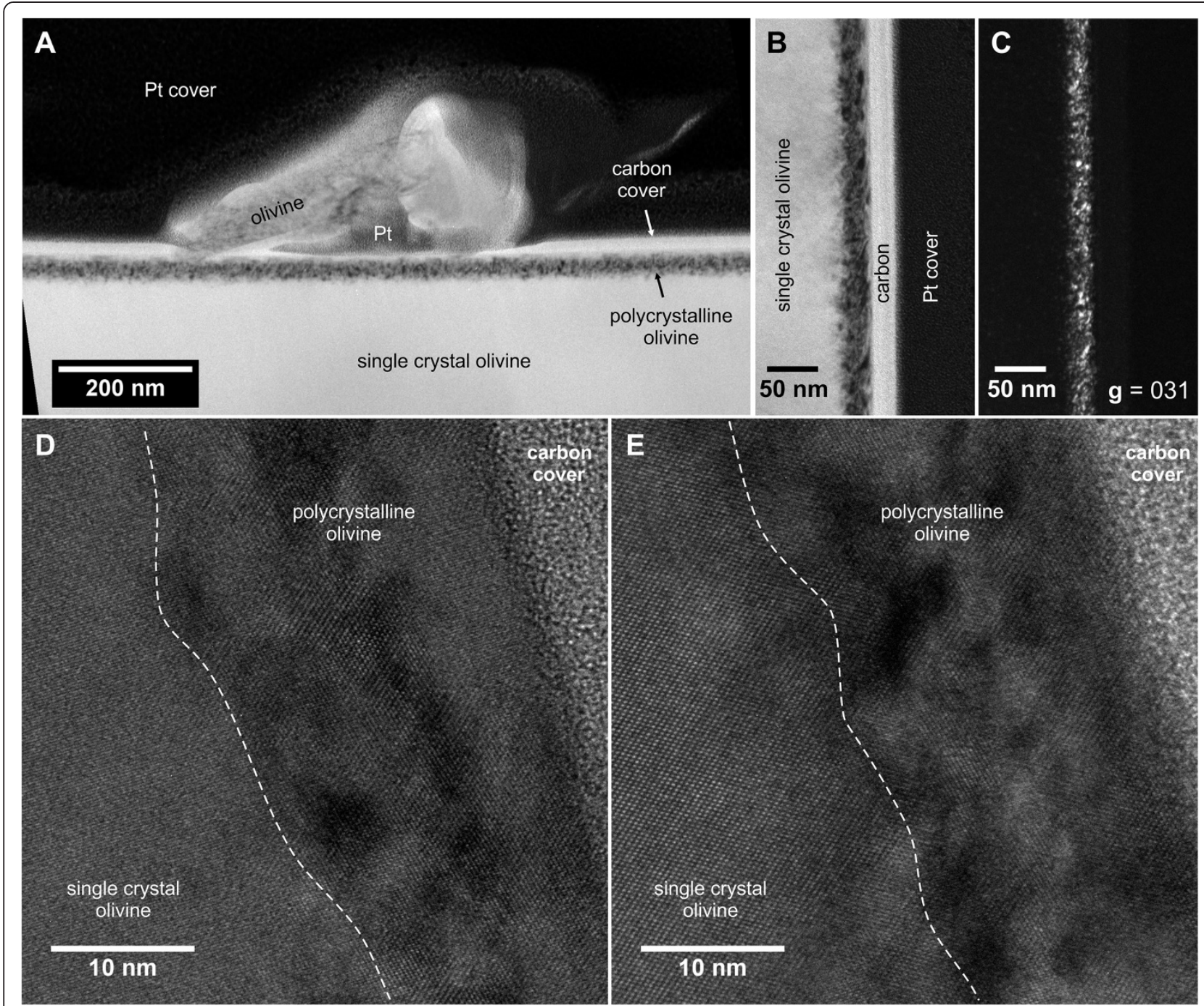

Figure 3 Polynanocrystalline olivine rims on the front surface of RA-QD02-0115. (A) TEM bright-field image showing the approximately 34 $\mathrm{nm}$ thick, strongly diffracting rim (subsample 05). The rim continues unchanged under a surficial olivine particle. (B) TEM bright-field image of the rim (subsample 04). The carbon and platinum coating were applied during FIB preparation. (C) Corresponding TEM dark-field image. Bright areas in the rim are crystalline, dark areas may be amorphous or crystalline with non-diffracting orientations (as in the bulk olivine). (D) HR-TEM image of the rim (subsample 04 ) along the foil normal $(\alpha=\beta=0)$, some crystallites of the rim are seen along $a<313>$ zone axis. (E) HR-TEM image (subsample 04, slightly different location) along a $<313>$ zone axis of the bulk olivine ( $\alpha=2.2, \beta=0.7)$. In (D) and (E), no large fractions of obviously amorphous regions can be seen, comparison of the tilted images suggests misorientations of the rim $s$ crystallites on the order of a few degrees.

no detectable amorphous or partially crystalline rim (Figure 4B).

The large troilite grain exposed on the front side of the particle and sectioned in subsample 02 (Figure 5) was found to have a very thin ( 4 to $8 \mathrm{~nm}$ ) surficial layer, which is composed of crystalline $\mathrm{FeS}$ domains that are structurally misaligned with the bulk of the inclusion. The angles of misalignment are on the order of several tens of degrees and, therefore, much larger than those observed in the olivine rim. Metallic iron was not observed in this rim.

\section{Discussion}

\section{Shock history of RA-QD02-0115}

Defect microstructures are generally scarce in all minerals composing particle RA-QD02-0115. This is contrary to particle RB-QD04-0042, which contains, for instance, olivine with high densities of straight [001] screw dislocations indicative of strong shock loading (Langenhorst et al. 2014). In RA-QD02-0115, the density of [001] dislocations is lower by at least one order of magnitude. Furthermore, the [001] dislocations are partially curved and do thus possess an edge component. 

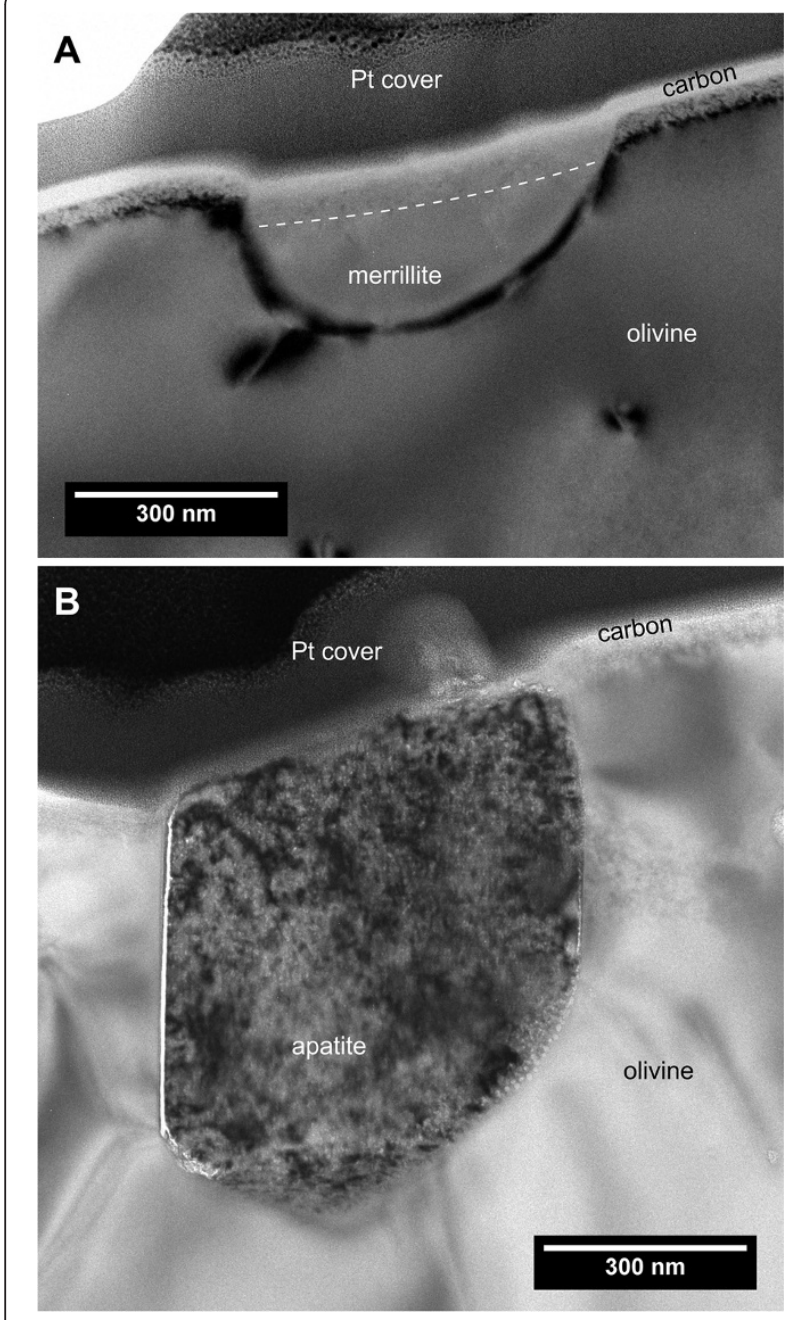

Figure 4 Phosphate grains exposed at the front surface of RA-QD02-0115. (A) TEM bright-field image of merrillite (subsample 04) along the foil normal. A weak spotting can be seen in the upper $50 \mathrm{~nm}$ (dashed line). (B) TEM bright-field image of apatite (subsample 04) along zone axis [100]. No rim structure is visible.

This observation is not consistent with a direct formation of the observed dislocations by strong shock deformation because the edge component of [001] dislocations in shocked olivine is distinctly faster than the screw component and would thus disappear during the passage of a shock wave (Langenhorst et al. 1999). Due to their longer Burgers vector relative to [100] dislocations, [001] dislocations are less favored to be activated, and high strains and strain rates are commonly assumed to be necessary for their mobilization. However, static experiments have shown that [001] dislocations can also be activated in a high-temperature regime (Nicolas and Poirier 1976). It appears thus to be a possible alternative to (weak) shock deformation that the dislocations were emitted and partially assembled into subgrain boundaries during plastic deformation while parent asteroid of Itokawa underwent thermal metamorphism.

\section{Space weathering of olivine in RA-QD02-0115}

The nanocrystalline olivine rim on the front side of the particle appears to be an unusual, or, as we rather think, a not much studied phenomenon. While the amorphization of olivine, pyroxenes, and plagioclase has been described in detail for multiple particles recovered from Itokawa (Noguchi et al. 2011, 2014; Thompson et al. 2014), polycrystalline rims containing nanometer-sized crystal domains and very little amorphous material have been recognized just recently (Keller and Berger 2014; this work). Keller and Berger (2014) estimated also a low density of solar flare tracks in a particle with a similar rim, which they interpret as an indication of a short exposure to the solar wind.

There may be several possible scenarios of how the rim observed on the surface of RA-QD02-0115 formed: (i) it represents an early stage of, nonetheless rapid, radiation damage due to the solar wind (Keller and Berger 2014), (ii) it has been recrystallized from a former, largely, or completely amorphous state, (iii) it formed by competing actions of irradiation damage and thermal annealing, or (iv) it formed by the re-deposition of sputtered or vaporized material, which we, however, exclude by the simple fact that we did not observe similar olivine layers on top of the other exposed minerals. The first three cases may also apply to the low density of solar flare tracks, either suggesting that tracks were not formed (i) or erased (ii, iii).

Early stage of space weathering Considering the first case, the observed rim may represent the very early stage of space weathering, in which the accumulated dose of radiation (mainly protons and helium at approximately 1 $\mathrm{keV} /$ nucleon) was not yet sufficient for substantial amorphization and the formation of $\mathrm{npFe} \mathrm{e}^{0}$, although it seem not fully clear whether $n p F e^{0}$ forms by in situ reduction of $\mathrm{Fe}^{2+}$ (e.g., Noguchi et al. 2011) or through vapor deposition processes (e.g., Sasaki et al. 2001). The low density of solar flare tracks also points to a short exposure to the solar wind. However, it seems surprising that a rather low dose alone will cause the quite large structural reorganization, in particular the rotation of crystalline domains, observed in the 30-nm-wide rim of RA-QD02-0115. The implantation of energetic particles into crystalline structures causes displacements of ions from structural lattice sites into interstitial positions, the sum of many such displacements eventually leads to amorphization. In situ irradiation experiments by Wang et al. (1999) using 1.5 $\mathrm{MeV} \mathrm{Kr}^{+}$ions directed at approximately $300 \mathrm{~nm}$ thick foils of $\mathrm{Fa}_{12}$ olivine showed that the disordering of the structure starts with the cations being preferentially knocked into interstitial positions, while the anion sublattice remains intact for a longer time at a given ion flux. Similar experiments by Christoffersen 

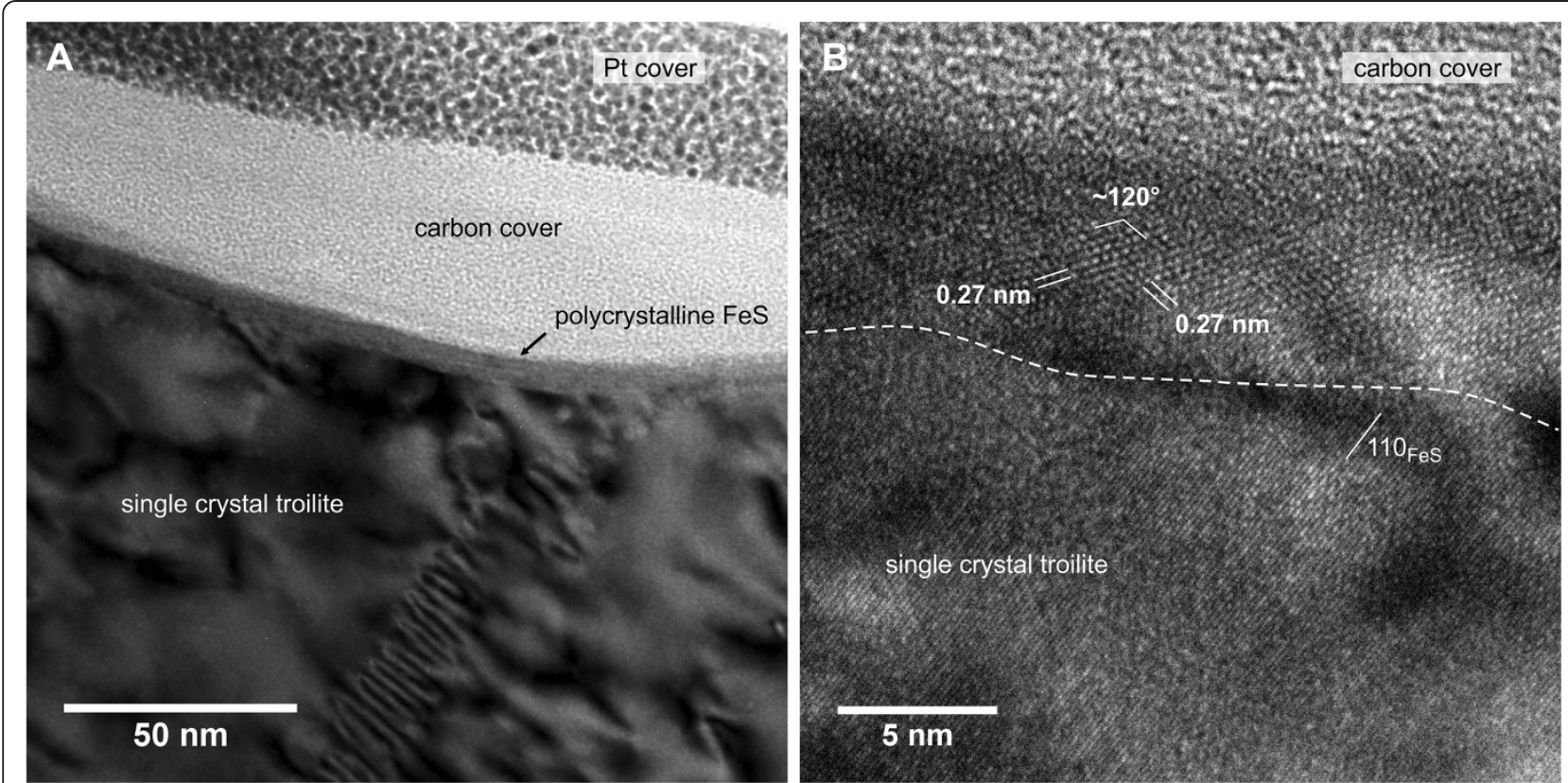

Figure 5 Troilite exposed at the front surface of RA-QD02-0115. (A) TEM bright-field image showing a 4 to $8 \mathrm{~nm}$ wide rim. (B) HR-TEM image of the polycrystalline sulfide rim showing crystallites that are consistent with hexagonal FeS.

and Keller (2011) confirmed that amorphization progresses by the formation of amorphous domains within a residual crystalline matrix that shows no crystallographic misorientations. Carrez et al. (2002) found similar amorphization effects and comparable critical fluences using 4 $\mathrm{keV} \mathrm{He}^{+}$ions directed at thin $\mathrm{Fa}_{10}$ olivine foils, suggesting that under the influence of the solar wind, the earliest loss of structural order would be accompanied by the preservation of the structural coherency of the cation sublattice. On the other hand, zircon studies have shown that at high irradiation doses, the rotation of remnant crystalline domains cause slight misorientations relative to the former bulk crystal (Murakami et al. 1991; Weber et al. 1994). This deformation is explained by stain fields resulting from advanced amorphization of surrounding material. However, in HRTEM images, this state is characterized by well-visible and, therefore, large amounts of amorphous domains. This is unlike the case of the olivine rims observed here. Similar textures have been reported from a zircon that had undergone significant annealing (Utsunomiya et al. 2004).

Recrystallization from an amorphous state If the rim had been recrystallized from an amorphous state, this would imply a thermal event that supplied sufficient activation energy for a transition back to an ordered crystal structure. The same applies for the recovery of damage along solar flare tracks, which would likely require less temperature or time, given their small size compared to a voluminous rim structure. Experimental investigations of the recrystallization of radiation-amorphized olivine by Djouadi et al. (2005) yielded activation energies for the process, which allow estimating the time scales required for the transition back to a crystalline state using an Avrami model of nucleation and crystal growth at a given temperature (Figure 6). Using a time exponent of one (i.e., a surface saturated nucleation with one-dimensional growth as expected for a thin amorphous film on a crystalline substrate) yields time scales larger than millions of years below temperatures of $600 \mathrm{~K}$. Rapid recrystallization of only a small fraction $(>5 \%)$ on the order of years to hours requires temperatures between 800 and 1,000 K. Given the current orbital parameters of Itokawa and using a simple gray body approximation of equilibrium between incident and radiated solar power (Harris and Lagerros 2002; bolometric albedo of 0.125 from Bernardi et al. 2009, infrared emissivity of 0.9 , zero thermal inertia, spherical shape), it is unlikely that its surface experiences temperatures in excess of $400 \mathrm{~K}$ even at perihelion under vertical illumination (note that the temperature measured at Muses Sea was $310 \mathrm{~K}$ at approximately $1 \mathrm{AU}$ distance, close to the perihelion of $0.95 \mathrm{AU}$; Yano et al. 2006). Because the dynamical orbital evolution of near-Earth asteroids with semi-major axes smaller than $2 \mathrm{AU}$ can lead to largely and chaotically variable orbital parameters (Morbidelli et al. 2002), it may be hypothesized that during its residency as a near-Earth asteroid (with typical lifetimes on the order of a few millions of years; Michel 


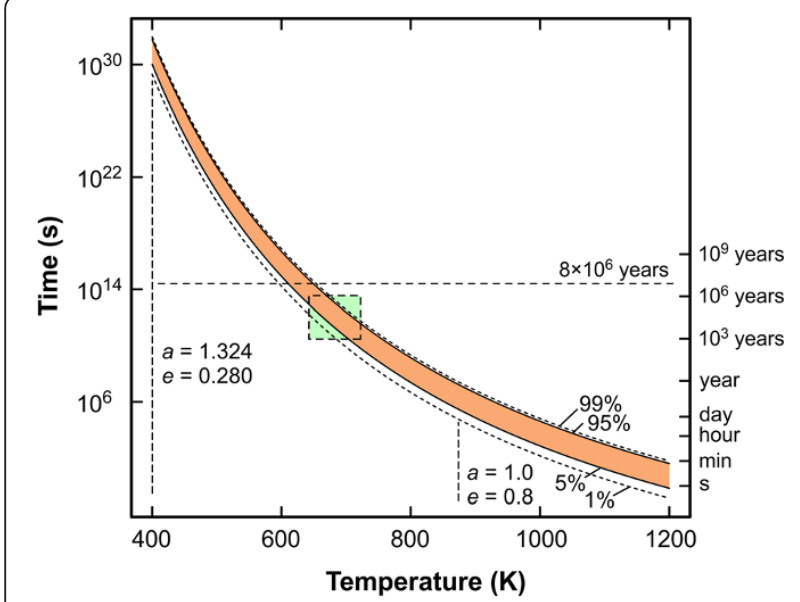

Figure 6 An Avrami recrystallization model (based on Djouadi et al. 2005). The time required for $1 \%, 5 \%, 95 \%$, and $99 \%$ recrystallization of a thin olivine film is shown. The vertical dashed lines give the maximum subsolar temperatures at perihelion for current Itokawa (left) and an assumed extreme orbit (right). The horizontal dashed line indicates the upper estimated exposure age of Itokawas surface (Nagao et al. 2011). The region between 650 and $700 \mathrm{~K}$ (dashed box) is particularly interesting because, here, recrystallization can occur on the time scales of thousands of years (see text).

and Yoshikawa 2005), Itokowa could have had closer perihelion approaches to the Sun and, therefore, may have experienced larger average and peak surface temperatures. In order to roughly estimate an upper limit of hypothetically attainable temperatures, we assume a quite extreme (but not unrealistic) orbit with a semi-major axis $a=1$ (currently $a=1.324$ ) and an eccentricity of $e=0.8$ (currently $e=0.28$ ). In this case, the perihelion passage (at 0.2 $\mathrm{AU})$ would not have led to peak surface temperatures above approximately $870 \mathrm{~K}$ and the average surface temperatures would have been significantly lower (note that there are Apollo class near-Earth asteroids with similar orbits, e.g., 1566 Icarus, 3200 Phaethon, 5786 Talos).

Based on the two bracketing cases of the current orbit and the extreme orbit, it seems at least possible that a near-Earth asteroid like 25143 Itokawa had experienced subsolar temperatures on the order of 650 to $700 \mathrm{~K}$ at some locations of its surface. If such an orbital episode lasted for several thousands of years, it might have had significant impact on the dynamical development of radiation-induced space weathering effects.

Competing damage and annealing We consider the subsequent recrystallization of an amorphized rim to be a rather unlikely extreme scenario. In such a case, there should probably be remnants of nanophase metallic iron (e.g., grown into larger particles), which would be expected to form during the progressed radiation damage before recrystallization. However, the synchronous action of radiation damage and some degree of thermal annealing could be a much more common scenario, given that the extreme case outlined above appears to be at least possible. Because the displacement of atoms by energetic particles competes with the relaxation and annealing of the disturbed structure via thermally activated atomic movements, temperature is an important factor in governing the accumulation of radiation damage. Wang et al. (1999) showed that for $\mathrm{Fa}_{12}$ olivine, the number of displacements per atom required for amorphization dramatically increased when the samples temperature approached approximately $500 \mathrm{~K}$. This is due to thermal annealing beginning to dominate over the rate of radiation-induced displacement. Although these experiments were done using a high-energy, high-flux $\mathrm{Kr}^{+}$ion beam (albeit on a thin sample, through which most ions were transmitted), it is clear that such competing effects must play a role during the exposure of minerals to the solar wind. Considering that the flux of incoming solar wind particles and, therefore, the rate of radiation damage are rather low compared to laboratory-based experiments, the rate of thermal annealing may be an even more important antagonist. One possible mechanism of formation of the nanocrystalline rim under the influence of annealing could be the formation of dislocation loops, which could aggregate into (poorly developed) low-angle grain boundaries. The formation of dislocation loops via irradiation has been observed in $\mathrm{Al}_{2} \mathrm{O}_{3}$ and $\mathrm{MgAl}_{2} \mathrm{O}_{4}$ crystals at $300 \mathrm{~K}$ (Zinkle and Pells 1998). Because olivines critical amorphization temperature is higher (approximately $500 \mathrm{~K}$; Wang et al. 1999), this process might only work at elevated temperatures.

The rates of damage and annealing depend strongly and with different scaling on the heliocentric distance of the irradiated body. On the scale of an asteroid, not all parts of the regolith would be expected to be affected similarly because the highest temperatures and ion fluxes would occur at subsolar locations, where the Sun is directly overhead and where minimal topographic shadowing effects occur. It might be expected that only few locations on an asteroid would statistically experience large above-the-average temperatures and, therefore, depending on the regolith mixing rate, only a very low fraction of the regolith grains would record an unusual temperature history.

Aside from the static effect of temperature, also, thermal cycling through diurnal and perennial temperature variations may influence the early development of amorphous rims. Because the amorphization is expected to be associated with an increase of the molar volume of the amorphous material (approximately 9.6\% for melt-quenched, vitreous $\mathrm{Mg}_{2} \mathrm{SiO}_{4}$ relative to forsterite; Kohara et al. 2004), the mechanical stress resulting from the formation of amorphous domains may be relieved by deformation 
involving the rotation of the remaining crystalline domains within the less rigid amorphous matrix. This deformation could potentially be intensified by the differential thermal expansion of the crystalline and amorphous domains and the crystalline substrate, if the amplitude of temperature variations was sufficiently high. Because the amount of amorphous material in the olivine rim of RA-QD02-0115 appears to be very low and there is no experimental evidence that such a process would work, we are cautious that thermal cycling alone could be responsible for the polycrystalline development of the rim during progressive radiation damage. We rather favor a contribution from thermally activated recrystallization after or dynamical annealing during exposure. The lack of $\mathrm{npFe}^{0}$ in the rim, given that it may form through in situ reduction of $\mathrm{Fe}^{2+}$, may be explained by a low dose of administered protons or the loss of $\mathrm{H}_{2}$ during an exposure at elevated temperatures, which prevented the reduction of iron (the reaction $2 \mathrm{H} \leftrightarrow \mathrm{H}_{2}$ would compete with the reaction $\mathrm{FeO}+2 \mathrm{H} \leftrightarrow$ $\left.\mathrm{Fe}^{0}+\mathrm{H}_{2} \mathrm{O}\right)$.

Elevated temperatures during exposure to the solar wind would probably have also profound effects on the retention and the composition of implanted noble gases. Diffusion would lead to a preferential loss of He relative to the heavier noble gases. Nagao et al. (2011) report a distinctly lower ${ }^{4} \mathrm{He} /{ }^{20} \mathrm{Ne}$ ratio in Hayabusa samples relative to the solar wind, which they interpret to be the result of mechanical erosion of fragile and easily removed space weathered rims and less due to diffusion. Diffusion would decrease the ${ }^{3} \mathrm{He} /{ }^{4} \mathrm{He}$ ration below that of the solar wind, which is not observed (Nagao et al. 2011). After gas loss or low accumulation of noble gases during an episode of elevated surface temperatures, replenishing of the lowtemperature (shallowly implanted) noble gas contents may occur rapidly on time scales of a few hundred years. Since the thermal processing and irradiation strongly depend on the location of the grains within the regolith and its location on the asteroid, it is difficult to correlate structural effects with noble gas contents not measured on the same grains.

\section{Space weathering of phosphates and troilite in RA-QD02-0115}

Due to the specific FIB targeting of the two phosphate grains exposed on the front side of particle RA-QD020115 (subsample 04), we can directly compare the exposure effects on merrillite and Cl-bearing apatite with the adjacent olivine. No detectable radiation damage in the apatite suggests a higher resistance against amorphization due to more rapid annealing of the radiation damage. Irradiation experiments with $1.5 \mathrm{MeV} \mathrm{Kr}^{+}$ions conducted by Wang et al. (1994) under comparable conditions to the experiments of Wang et al. (1999) showed that the critical, upper temperature of amorphization of $\mathrm{Fa}_{12}$ olivine and fluorapatite are very similar. Increasing
Fe contents of olivine shifts this temperature to higher values (i.e., the structure becomes more vulnerable), and therefore, the observed difference between the two phases in our sample is probably due to the olivines higher Fe content. A higher recovery rate of the $\mathrm{Cl}$-rich apatite relative to fluorapatite is rather unlikely as studies on fission track annealing in apatite have shown a tendency of reduced recovery in Cl-rich apatites (e.g., Ketcham et al. 1999). Compared to the apatite, merrillite appears to be somewhat more susceptible to radiation damage by the solar wind given its marginally discernable rim structure observed in our sample.

The thin, polycrystalline rim found on the troilite surface suggests that the sulfide has a lower depth penetration range of incoming ions due to its moderately higher density compared to the ferromagnesian silicates. As shown by Keller and Rahman (2011), the sputtering yield of ions impinging troilite may be several times higher compared to the silicates, suggesting that the thin rim may be explained in part by a significant mass loss during irradiation.

\section{Conclusions}

Our results show that FIB preparation of small particles can be used to specifically cross-section selected surface regions of interest. The composition of the olivine and the generally very $\mathrm{Ni}$-rich metal assemblages indicate an LL-type origin also for particle RA-QD02-0115. Unlike the effects seen in particle RB-QD04-0042, we found no compelling microstructural evidence for recent shock mechanical modifications via small-scale impacts. Radiation damage by energetic ions of the solar wind occurs on one side of particle RA-QD02-0115 and can be observed in olivine, troilite, and weakly in merrillite. Clbearing apatite and metal have not sustained detectable damage. The unusual polycrystalline nature of the olivine rim, containing nanometer-sized domains tilted by a few degrees, may be explained by annealing effects at asteroidal surface temperatures larger than expected and observed at the current orbital parameters of Itokawa. We acknowledge that it would be a rather long shot to ascribe the apparently unusual surface state of RAQD02-0115 to be connected to a dynamical evolution of its parent asteroid, in particular, because we would expect more particles in the Hayabusa collection to show signs of annealing. After all, RA-QD02-0115 may show the normal, early stages of radiation damage, which we just do not know well enough. However, RA-QD02-0115 may have resided at an unusually hot and statistically rare location and there might be other particles that may show more pronounced effects of thermal annealing. The possibility of a former orbit closer to the Sun appears not to be too unrealistic and the thermal consequences of such a scenario appear worthwhile to be 
investigated in more detail, in particular with respect to the retention of implanted noble gases by correlating space weathering effects with gas contents of the same grains. Contrary to the moon, space weathering on nearEarth asteroids may be strongly linked to the dynamical evolution of their orbits. Specific selection of future sampling sites may provide clues of the orbital and thermal histories of these asteroids and their surfaces.

\section{Abbreviations}

EDX: energy-dispersive X-ray spectrometry; FIB: focused ion beam; GP: glycol phthalate; HR-TEM: high-resolution transmission electron microscopy; SAED: selected area electron diffraction; $\mathrm{npFe}^{0}$ : nanoparticulate metallic iron; SEM: scanning electron microscope/microscopy; TEM: transmission electron microscope/microscopy; WDX-EPMA: wavelength-dispersive X-ray electron probe microanalysis.

\section{Competing interests}

The authors declare that they have no competing interests.

\section{Authors contributions}

$\mathrm{DH}$ conducted the FIB preparation of the sample. DH and FL carried out the data collection and interpretation. DH calculated the Avrami model and wrote the manuscript with contributions from FL. Both authors read and approved the final manuscript.

\section{Acknowledgements}

We gratefully acknowledge JAXA for the allocation of two Hayabusa samples in the framework of the first announcement of opportunity for Hayabusa sample investigations. This research received funding by the Deutsche Forschungsgemeinschaft DFG (grant LA 830/14-1 to FL). We acknowledge the constructive and helpful comments of two anonymous reviewers.

\section{Received: 30 April 2014 Accepted: 26 November 2014}

\section{Published online: 16 December 2014}

\section{References}

Bernardi F, Micheli M, Tholen DJ (2009) Absolute magnitude and slope parameter G calibration of asteroid 25143 Itokawa. Meteorit Planet Sci 44:1849 1852

Binzel RP, Bus SJ, Burbine TH, Sunshine JM (1996) Spectral properties of near-Earth asteroids: evidence for sources of ordinary chondrite meteorites. Science 373:946 948

Carrez P, Demyk K, Cordier P, Gengembre L, Grimblot J, D hendecourt L, Jones AP, Leroux H (2002) Low-energy helium ion irradiation-induced amorphization and chemical changes in olivine: insights for silicate dust evolution in the interstellar medium. Meteori Planet Sci 37:1599 1614

Chapman CR (2004) Space weathering of asteroid surfaces. Ann Rev Earth Planet Sci 32:539 567

Christoffersen R, Keller LP (2011) Space radiation processing of sulfides and silicates in primitive solar system materials: comparative insights from in situ TEM ion irradiation experiments. Meteorit Planet Sci 46:950 969

Djouadi Z, D Hendecourt L, Leroux H, Jones AP, Borg J, Deboffle D, Chauvin N (2005) First determination of the (re)crystallization activation energy of an irradiated olivine-type silicate. Astron Astrophys 440:179 184

Harries D, Langenhorst F (2014) Haxonite from 25143 Itokawa and its implications for metamorphic parent body fluids. Meteorit Planet Sci 49:A157

Harris AW, Lagerros JSV (2002) Asteroids in the thermal infrared. In: Bottke WF (ed) Asteroids III. University of Arizona Press

Keller LP, McKay DS (1997) The nature and origin of rims on lunar soil grains. Geochim Cosmochim Acta 61:2331 2341

Keller LP, Rahman Z (2011) Irradiation of FeS: relative sputtering rates of troilite and Mg silicates. Meteorit Planet Sci 46:A121

Keller LP, Berger EL (2014) A transmission electron microscope study of Itokawa regolith grains. Earth Planets Space, doi:10.1186/1880-5981-66-71

Ketcham RA, Donelick RA, Carlson WD (1999) Variability of apatite fission-track annealing kinetics: III. Extrapolation to geological time scales. Am Mineral 84:1235 1255
Kohara S, Suzuya K, Takeuchi K, Loong C-K, Grimsditch M, Weber JKR, Tangeman JA, Key TS (2004) Glass formation at the limit of insufficient network formers. Science 303:1649 1652

Langenhorst F, Harries D, Pollok K, van Aken PA (2014) Mineralogy and defect microstructure of an olivine-dominated Itokawa dust particle: evidence for shock metamorphism, collisional fragmentation, and LL chondrite origin. Earth Planets Space, doi:10.1186/1880-5981-66-118

Langenhorst F, Boustie M, Migault A, Romain JP (1999) Laser shock experiments with nanoseconds pulses: a new tool for the reproduction of shock defects in olivine. Earth Planet Sci Lett 173:333 342

Michel P, Yoshikawa M (2005) Earth impact probability of the Asteroid (25143) Itokawa to be sampled by the spacecraft Hayabusa. Icarus 179:291 296

Morbidelli A, Bottke WF, Froeschle C, Michel P (2002) Origin and evolution of near-Earth objects. In: Bottke WF (ed) Asteroids III. University of Arizona Press

Murakami T, Chakoumakos BC, Ewing RC, Lumpkin GR, Weber WJ (1991) Alpha-decay event damage in zircon. Am Mineral 76:1510 1532

Nagao K, Okazaki R, Nakamura T, Miura YN, Osawa T, Bajo K, Matsuda S, Ebihara M, Ireland TR, Kitajima F, Naraoka H, Noguchi T, Tsuchiyama A, Yurimoto H, Zolensky ME, Uesugi M, Shirai K, Abe M, Yada T, Ishibashi Y, Fujimura A, Mukai T, Ueno M, Okada T, Yoshikawa M, Kawaguchi J (2011) Irradiation history of Itokawa regolith material deduced from noble gases in the Hayabusa samples. Science 333:1128 1131

Nakamura T, Noguchi T, Tanaka M, Zolensky ME, Kimura M, Tsuchiyama A, Nakato A, Ogami T, Ishida H, Uesugi M, Yada T, Shirai K, Fujimura A, Okazaki R, Sandford SA, Ishibashi Y, Abe M, Okada T, Ueno M, Mukai T, Yoshikawa M, Kawaguchi J (2011) Itokawa dust particles: a direct link between S-type asteroids and ordinary chondrites. Science 333:1113 1116

Nakamura E, Makishima A, Moriguti T, Kobayashi K, Tanaka R, Kunihiro T, Tsujimori T, Sakaguchi C, Kitagawa H, Ota T, Yachi Y, Yada T, Abe M, Fujimura A, Ueno M, Mukai T, Yoshikawa M, Kawaguchi J (2012) Space environment of an asteroid preserved on micrograins returned by the Hayabusa spacecraft. Proc Nat Acad Sci USA 109:E624 E629

Nicolas A, Poirier JP (1976) Crystalline Plasticity and Solid State Flow in Metamorphic Rocks. Wiley, London

Noguchi T, Nakamura T, Kimura M, Zolensky ME, Tanaka M, Hashimoto T, Konno M, Nakato A, Ogami T, Fujimura A, Abe M, Yada T, Mukai T, Ueno M, Okada T, Shirai K, Ishibashi Y, Okazaki R (2011) Incipient space weathering observed on the surface of Itokawa dust particles. Science 333:1121 1125

Noguchi T, Kimura M, Hashimoto T, Konno M, Nakamura T, Zolensky ME, Okazaki R, Tanaka M, Tsuchiyama A, Nakato A, Ogami T, Ishida H, Sagae R, Tsujimoto S, Matsumoto T, Matsuno J, Fujimura A, Abe M, Yada T, Mukai T, Ueno M, Okada T, Shirai K, Ishibashi Y (2014) Space weathered rims found on the surfaces of the Itokawa dust particles. Meteorit Planet Sci 49:188 214

Pieters CM, Taylor LA, Noble SK, Keller LP, Hapke B, Morris RV, Allen CC, McKay DS, Wentworth S (2000) Space weathering on airless bodies: resolving a mystery with lunar samples. Meteorit Planet Sci 35:1101 1107

Sasaki S, Nakamura K, Hamabe Y, Kurahashi E, Hiroi T (2001) Production of iron nanoparticles by laser irradiation in a simulation of lunar-like space weathering. Nature 410:555 557

Thompson MS, Christoffersen R, Zega TJ, Keller LP (2014) Microchemical and structural evidence for space weathering in soils from asteroid Itokawa. Earth Planets Space, doi:10.1186/1880-5981-66-89

Utsunomiya S, Palenik CS, Valley JW, Cavosie AJ, Wilde SA, Ewing RC (2004) Nanoscale occurrence of Pb in an Archean zircon. Geochim Cosmochim Acta 68:4679 4686

Van Cappellen E, Doukhan JC (1994) Quantitative X-ray microanalysis of ionic compounds. Ultramiscroscopy 53:343 349

Wang LM, Miller ML, Ewing RC (1993) HRTEM study of displacement cascade damage in krypton-ion-irradiated silicate olivine. Ultramicroscopy 51:339 347

Wang LM, Cameron M, Weber JM, Crowley KD, Ewing RC (1994) In situ TEM observation of radiation induced amorphization of crystals with apatite structure. In: Brown PW, Constantz B (eds) Hydroxyapatite and Related Materials. CRC Press, Boca Raton

Wang LM, Gong W, Wang S, Ewing RC (1999) Comparison of ion-beam irradiation effects in $\mathrm{X}_{2} \mathrm{YO}_{4}$ compounds. J Am Ceram Soc 82:3321 3329

Weber WJ, Ewing RC, Wang LM (1994) The radiation-induced crystalline-toamorphous transition in zircon. J Mater Res 9:688 698

Yada T, Fujimura A, Abe M, Nakamura T, Noguchi T, Okazaki R, Nagao K, Ishibashi Y, Shirai K, Zolensky ME, Sandford S, Okada T, Uesugi M, Karouji Y, Ogawa M, Yakame S, Ueno M, Mukai T, Yoshikawa M, Kawaguchi J (2014) Hayabusa-returned sample curation in the Planetary Material Sample Curation Facility of JAXA. Meteorit Planet Sci 49:135153 
Yano H, Kubota T, Miyamoto H, Okada T, Scheeres D, Takagi Y, Yoshida K, Abe M, Abe S, Barnouin-Jha O, Fujiwara A, Hasegawa S, Hashimoto T, Ishiguro M, Kato M, Kawaguchi J, Mukai T, Saito J, Sasaki S, Yoshikawa M (2006) Touchdown of the Hayabusa Spacecraft at the Muses Sea on Itokawa. Science 312:1350 1353

Zinkle SJ, Pells GP (1998) Microstructure of $\mathrm{Al}_{2} \mathrm{O}_{3}$ and $\mathrm{MgAl}_{2} \mathrm{O}_{4}$ irradiated at low temperatures. J Nucl Mater 253:120 132

doi:10.1186/s40623-014-0163-1

Cite this article as: Harries and Langenhorst: The mineralogy and space weathering of a regolith grain from 25143 Itokawa and the possibility of annealed solar wind damage. Earth, Planets and Space 2014 66:163.

\section{Submit your manuscript to a SpringerOpen ${ }^{\circ}$} journal and bene $\otimes t$ from:

- Convenient online submission

- Rigorous peer review

- Immediate publication on acceptance

- Open access: articles freely available online

- High visibility within the 区eld

- Retaining the copyright to your article

Submit your next manuscript at $\gg$ springeropen.com 